\title{
Analysis of Lamin B1, Vimentin and Anti-Ku86 as Prospective Biomarkers of Hepatocellular Carcinoma in Patients with Hepatitis C Virus Infection
}

\author{
Naglaa K. Idriss ${ }^{a} \quad$ Michel Fakhry ${ }^{a} \quad$ Hala M. Imam ${ }^{b}$ Fatema A. Abd-Elmoez \\ Hossam Abdelewahab ${ }^{b}$ Lobna Abdel-Wahid ${ }^{b}$ Wael A. Abbas $^{b}$ \\ Mohamed A.A. Abozaid ${ }^{b}$ Zain Sayed $^{b} \quad$ Ahmed M. Ashmawy ${ }^{b}$ \\ aDepartment of Medical Biochemistry, Faculty of Medicine, Assiut University, Assiut, Egypt, \\ ${ }^{b}$ Department of Internal Medicine, Faculty of Medicine, Assiut University, Assiut, Egypt
}

\section{Key Words}

Chronic hepatitis $C \cdot$ Hepatocellular carcinoma $・$ Vimentin $\bullet$ LaminB1 Antiku 86

\begin{abstract}
Background/Aims: Hepatocellular carcinoma (HCC) is the fifth most common malignancy and most frequently develops in patients with cirrhosis. Surveillance strategies are recommended in high-risk groups because early detection of small lesions improves the likelihood of curative treatment. This study investigated the prospective clinical significance of serum levels of anti-Ku86 and plasma levels of lamin B1and vimentin as early markers of HCC. Methods: We recruited 74 patients at Assiut University Hospital-37 with HCC and 37 with chronic liver disease (liver cirrhosis patients)—and 36 age- and sex-matched healthy controls. Lamin $B 1$ and vimentin mRNA expression levels were evaluated by reverse transcription-PCR and serum levels of anti-Ku86 were measured by enzyme-linked immunosorbent assay. Results: Compared with liver disease patients and controls, HCC patients showed higher levels of lamin B1 mRNA (sensitivity, 96\%; specificity, 65\%), vimentin mRNA (sensitivity, 94\%; specificity, 92\%), and anti-Ku86 (sensitivity, 94\%; specificity, 80\%). LaminB1 levels were significantly higher in patients with a tumor size $<2 \mathrm{~cm}$ than in patients with tumors $2-5 \mathrm{~cm}$ and $>5 \mathrm{~cm}$ in size. Lamin $\mathrm{B} 1 \mathrm{had}$ significant positive correlations with alpha-fetoprotein (AFP) $(P=0.034)$ and anti-Ku86 $(P=0.002)$. Receiver operating characteristic curves for differentiating HCCfrom liver cirrhosis revealed a higher area under the curve(AUC).for vimentin than for AFP, lamin B1, and anti-Ku86 for the diagnosis of HCC $(P<0.001)$. Conclusion: Circulating levels of anti-Ku86, lamin B1, and vimentin might be potential surrogate markers of HCC, either alone or in combination with AFP. However, independent and discriminative serological biomarkers with higher sensitivity and specificity are still needed for the early detection of HCC.




\section{Introduction}

Hepatocellular carcinoma (HCC) is the third leading cause of cancer-related death, after lung and stomach cancer [1]. The main challenge of this cancer is the difficulty of early diagnosis. The chief causes of HCC are liver fibrosis or cirrhosis from chronic viral infections. Because cases tend to arise from proceeding pathologies, biomarker surveillance in high-risk individuals is an indispensable tool for achieving earlier detection and improved outcomes of HCC [1-3]. Multiple risk factors can trigger HCC development and progression, including viral hepatitis $\mathrm{B}$ and $\mathrm{C}$ infection, chronic alcohol consumption, metabolic disorders, and age [2].

Because of the high level of viral exposure in Egypt, more studies are needed to determine the risk factors for HCC and how to prevent it. In addition, there is evidence of increased liver cancer incidence in Egypt [4] and Egypt has one of the highest levels of hepatitis C virus infection in the world [5].

Ultrasonography is endorsed by all current guidelines for detecting HCC, although novel procedures are anticipated to increase the detection rate of the disease. Of these methods, serum alpha-fetoprotein (AFP)has been the most widely studied, but its utility is controversial. Although its specificity is good (85\%-90\%), it has low sensitivity $(18 \%-60 \%)$ [6]. In addition, it has recently been shown that most small HCC nodules do not increase AFP levels, decreasing the sensitivity of AFP for tumors smaller than $3 \mathrm{~cm}$ to just $25 \%$ [7]. Accordingly, new cost-effective and sensitive biomarkers for improving investigative accuracy are vital to complement current imaging tools for detecting HCC at early stages, when curative interventions can be applied [8].

Lamins are major constituents of intermediate filaments proteins, now classified as type $\mathrm{V}$ intermediate filament proteins [9]. Lamins have two subtypes, A-type and B-type. A-type lamins together with lamins $\mathrm{A}, \mathrm{C}, \mathrm{A} 10$, and $\mathrm{C} 2$ are merged products of the LMNA gene. The B-type lamins (lamins B1 [LMNB1], B2, and B3) are the products of two distinct genes $[9,10]$. LMNB1 is linked to cell proliferation through multiple mechanisms. It gives structural support to the nuclear envelope and participants in control of gene expression via the regulation of chromatin, DNA replication, repair, and transcription [11]. Many reports have noted that changes in the expression levels of LMNB1 are a sign of replicative and oncogene-induced cellular senescence [12]. Indeed, serum lamin concentrations are increased in metastatic cancers of various origins, such as gastric adenocarcinoma, HCC, colorectal cancer, and epithelial ovarian tumor [13-15].

Vimentin is classified as a type III intermediate filament protein. Its function is to maintain cellular integrity and protect the cell against stress. It is the major cytoskeletal component of mesenchymal cells [16]. Vimentin also plays a significant role in cell shape maintenance and in stabilizing cytoskeletal interactions [17-19]. Its overexpression has been found in various cancers, including prostate cancer, gastrointestinal tumors, CNS tumors, breast cancer, and malignant melanoma [18].

Many proteins are known to be overexpressed in HCC tissues. Consequently, serum autoantibodies to these overexpressed proteins are detectable in HCC patients. Ku is one such protein overexpressed in HCC. The Ku complex is composed of two subunits of 70 and 86 $\mathrm{kDa}$, which are accordingly named Ku70 and Ku86. Ku70 and Ku86 are the regulatory regions of a DNA-dependent protein kinase involved in DNA double-strand break repair, telomere length maintenance, cell cycle progression, transcriptional regulation, and other biological processes [20, 21]. Ku86 appears to trigger antigenicity relatively early in tumorigenesis. Thus, the serum levels of Ku86 antibodies are significantly higher in HCC patients than in liver cirrhosis patients [20-23].

In the present study, we assessed the plasma levels of LMNB1 and vimentin mRNA as well as the serum levels of anti-Ku86 in the peripheral blood of HCC patients and examined the relationship of these potential biomarkers with the pathogenesis of HCC. 


\section{Cellular Physiology Cell Physiol Biochem 2019;52:595-605 \\ \begin{tabular}{l|l} 
and: $10.33594 / 000000042$ & O 2019 The Author(s). Published by \\
Cell Physiol Biochem Press GmbH\&Co. KC
\end{tabular} \\ Idriss et al.: Genetic Indices in Patients with Hepatocellular Carcinoma}

\section{Materials and Methods}

\section{Patients}

In total, 37 patients withHCC and 37 patients with chronic liver disease (CLD)were recruited, as well as 36 age- and sex-matched healthy controls (HCs). The patients were selected from the outpatient clinic and inpatient department of Rajhy Liver Hospital and the Gastroenterology and Hepatology Unit in the internal medicine department of Assuit University Hospital.Epidemiological data were collected and risk factors were examined through medical records and an in-person questionnaire.

The etiology of the underlying CLD was ascribed to hepatitis $C$ virus based on the detection of hepatitis C virus antibody/RNA in the serum and to hepatitis B viru sbased on the detection of hepatitis B surface antigen in serum. The CLD group comprised patients with no evidence of hepatic mass lesions suspicious for HCC on ultrasound, computed tomography (CT), or magnetic resonance imaging (MRI). A diagnosis of HCC required at least two liver imaging outcomes indicating a mass lesion with characteristics similar to those of HCC. Patients with typical HCC displayed hypervascularity in the arterial phase and washout of contrast medium in the portal venous phase. Although ultrasonography is widely used for tumor surveillance in patients at risk of HCC, lesion classification was based on either a biopsy specimen or, in selected cases, on highly specific CT/MRI findings. The BCLC system was used for all HCC patients. The system identifies patients with early HCC who may benefit from curative therapies (stage 0 and A), those in the intermediate (stage B) and advanced (stage C) stages who might benefit from palliative treatments, and those with a very poor life expectancy (stage D) who often have contraindications to invasive treatment. Pathological diagnosis of HCC requires a biopsy of the tumor or a resection specimen, which should contain surrounding non-tumoral parenchyma evaluated according to appropriate CT or MRI characteristics. The Child-Pugh classification was used for all patients. Tumor stage was assessed using the BCLC staging system, which is based on tumor number, size, vascular invasion, and metastasis [24].

Exclusion criteria were as follows: significant valvular heart disease, previous coronary artery bypass surgery, primary angioplasty for ST-segment elevation acute myocardial infarction, infection or fever, recent (<3 months) ischemic stroke, chronic and systemic illnesses including renal failure (on supportive therapy), and inflammatory connective tissue disease, inflammatory bowel disease, any oral steroid intake, and/or hormone replacement therapy.

All participants underwent complete history taking, complete clinical examination, abdominal ultrasonography, and abdominal MRI (for patients with cirrhosis).

The study protocol was approved by the ethics committee of Assiut University and all participants provided written informed consent before taking part in the study.

\section{Laboratory tests}

Routine laboratory tests that included complete blood counts were performed on an automated hematology analyzer(KX-21, Sysmex, Kobe, Japan). Tests of liver function included analyses ofserum bilirubin, urine bilirubin, direct bilirubin, total bilirubin, urobilinogen, alanine transaminase, aspartate transaminase, albumin, total protein, and alkaline phosphate.Tests were also performed to detect injury to hepatocytes (serum enzyme tests) such as aminotransferases and alkaline phosphatase on an autoanalyzer (Integra 400, RocheDiagnostics, Mannheim, Germany). Hepatitis B surface antigen and hepatitis C virus antibody tests were performed using electrochemiluminescence immunoassay (ECLIA;Elecsys 2010, RocheDiagnostics), and serum AFP was meausured (Elecsys 2010, RocheDiagnostics).

\section{Biochemical tests}

We collected $5 \mathrm{ml}$ of blood from each participant; $3 \mathrm{ml}$ was placed in EDTA tubes and stored at $-70^{\circ} \mathrm{C}$ to allow batch analysis by real-time polymerase chain reaction (RT-PCR)while $2 \mathrm{ml}$ was placed in plain tubes and allowed to coagulate. The latter tubes werecentrifuged at 3,000 rpm for $20 \mathrm{~min}$ at $4^{\circ} \mathrm{C}$ to isolate the serum, which was stored at $-70^{\circ} \mathrm{C}$ to allow batch analysis by enzyme-linked immunosorbent assay (ELISA). We measured the relative levels of LMNB1 and vimentin mRNA in the plasma usingRT-PCR (see below) and those of anti-Ku86 in the serum using ELISA (Ku86 ELISAkit); The level of the Human Anti 86 kDa subunit of Ku antibody wasmeasured using a human Ku antibody (Ku86) ELISA kit according to the manufacturer's protocol [(Catalog \#MBS749325,(MyBioSource's Products)]. 


\section{Genetic analysis}

Total RNA was extracted from between $0.5 \mathrm{ml}$ and $1.0 \mathrm{ml}$ of whole blood using the RNeasy Mini Kit 250 (Cat.no.: 74106; Qiagen, Venlo, Netherlands), followed by a SensiFAST ${ }^{\text {TM }}$ SYBR® Hi-ROX One-Step Kit for cDNA synthesis and highlysensitive, reproducible RT-PCR detection in a single tube.The reactions were performed in a thermal cycler (MJ Research, Inc., Waltham, MA). The thermal profile consisted of $2 \mathrm{~min}$ at $50{ }^{\circ} \mathrm{C}$ and 10 min at $95^{\circ} \mathrm{C}$, followed by 40 cycles of $15 \mathrm{~s}$ at $95^{\circ} \mathrm{C}$ and $1 \mathrm{~min}$ at $60^{\circ} \mathrm{C}$. Gene expression was normalized to that of GAPDH as a controland is reported as foldchange.We used $1 \mu \mathrm{M}$ of each forward and reverse primer specific for each target gene:

LMNB1, 5'TCGCAAAAGCATGTATGAAGA3'and5'CT CTACCAAGCGCGTTTCA-3';

Vim, 5'-GCTGCAGGCCCAGATTCA-3' and 5'-TTCATACTGCTGGCGCACAT-3';

and GAPDH, 5' -AGCCACATCGCTCAGACAC-3' and 5' GCCCAATACGACCAAATCC-3'.

The reverse transcription step was repeated using the housekeeping gene GAPDH. The amplified products were hybridized to SYBR green dye using 5'

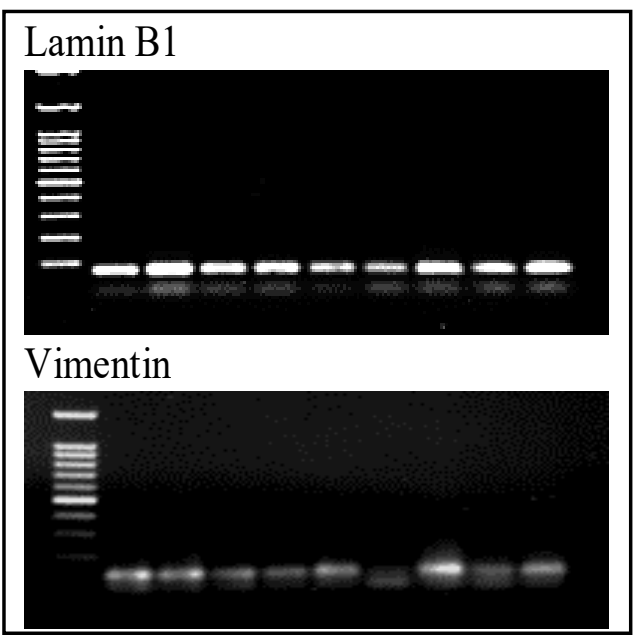

Fig. 1. Gel electrophoresis. PCR products were size-fractionated on $2 \%$ agarose gels and visualized under UV light. A, lamin B1 gene: Lanes 2-4, HCs; Lanes 5-7, CLD group ; and Lanes 8-10, HCC group. B, vimentin gene in HCs, CLD group, and HCC group (as above).

Hot FirepolEva Green qPCR Mix Plus (Cat. no.:08-25-0001; Solis BioDyne, Tartu, Estonia).

The mean expression levels of LMNB1 mRNA and vimentin mRNA relative to those of GAPDH were calculated using the quantitation-comparative $\mathrm{Ct}(\Delta \Delta \mathrm{Ct})$ method on an fluorescence quantitative PCR analyzer (ABI 7500, Applied Biosystems, Foster City, CA). After correction with the corrector sample, the relative expression levels were subjected to statistical analysis. Each RT-PCR assay was repeated twice and PCR products were confirmed by DNA sequencing and were size-fractionated on $2 \%$ agarosegels after ethidium bromide staining and visualized under UV light (Gel Electrophoresis, Fig. 1) (for supporting information see Supplementary Material - all supplementary material available online at www.cellphysiolbiochem.com).

\section{Statistical analysis}

Data were verified, coded by a researcher, and analyzed using SPSS version 21(SPSS Chicago, IL, USA). For descriptive statistics, means, standard deviations, medians, and percentages were calculated and analyzedusing the chi-square test, independent t-test, Mann-Whitney U test, ANOVA test, and Kruskal-Wallis test, as appropriate. Pearson correlation analysis was used to test the associations between variables. The associations between categorical variables and the final diagnosis of HCC was assessed via the chi-square test or Fisher exact test.Clinical and demographic factors with proven statistical significance in univariate analyses were included in multivariate logistic regression models. The receiver operating characteristic (ROC) curve was plotted for the tested HCC biomarkers, and the diagnostic accuracy measuresof the biomarkers were calculated (main validity measures). A $P$-value $\leq 0.05$ was considered significant.

\section{Results}

There were no significant differences in sociodemographic characteristics among the three groups, including age $(P>0.05)$ (Table 1$)$.

The laboratory findings of the three groups are shown in Table 2. The serum levels of aspartate transaminase were significantly higher in HCC patients than in CLD patients $(P<0.01)$. However, there were no significant differences between the HCC and CLD groups in terms of the other laboratory variables, including alanine transaminase, alkaline phosphatase, hemoglobin, platelets, bilirubin, albumin, and creatinine. 
AFP levels were significantly higher in the HCC group than in the CLD group $(P<0.05)$ and in the $\mathrm{HC}$ group $(P<0.001)$ (Table 3). LMNB1 mRNA levels were significantly higher in HCC patients than in CLD patients and HCs $(P<0.001)$. In addition, vimentin mRNA levels were significantly higher in the HCC group than in the other groups $(P<0.001$; Table 3). Serum levels of anti-Ku86weresignificantly higher in the HCC group than in the CLD group $(P<0.001$; Table 4, Fig. 2$)$.

Regarding the relationship between tumor markers and tumor size, there was no significant relationship of AFP, vimentin, or anti-Ku86 with tumor size. However, there was a significant positive correlation between LMNB1and a tumor size less than $2 \mathrm{~cm}$ in HCC patients $(P=0.014)$ (Table 5, Fig. 3).

Likewise, there was a significant decrease in vimentin mRNA levels in patients with portal vein thrombosis $\quad(P<0.05)$. Nevertheless, there was no significant relationship of the serum levels of AFP, LMNB1, and anti-Ku86 with portal vein thrombosis (Table 6, Fig. 4).

Univariate analysis of tumor markers and tumor characteristics showed that vimentin levels were significantly lower in patients with portal vein thrombosis $(P=0.014)$. However, there were no significant correlations of tumor size and tumor number with other tumor markers (AFP, LMNB1, and anti-Ku86) (Table 7).

There was a significant positive correlation between LMNB1 and AFP ( $\mathrm{r}=0.26, P=0.034)$. In addition, there was a significant positive correlation between LMNB1and anti-Ku86 $(\mathrm{r}=0.40, P=0.002)$ (Table 8, Fig. 3 and 4). However, no correlation was noted between the markers and tumor size (Fig. 5)

The ROC curves of AFP, LMNB1, vimentin, and anti-Ku86 for differentiating HCC from CLD are shown in Fig. 6 and 7. The highest areas under the curve (AUCs) for distinguishing HCC and CLD were 0.878, 0.837, 0.897, and 0.876 for AFP, LMNB1, vimentin, and anti-
Table 1. Sociodemographic data of the study groups. * ANOVA was used to compare the mean difference between groups. **Student's t-test test was used to compare the mean difference between the two groups. ${ }^{* * *}$ Chi-square test was used to compare the percentages between groups. "Z-test was used to compare the improvement percentages. $\$$ Mean \pm SD

\begin{tabular}{|c|c|c|c|c|}
\hline Parameter & $\begin{array}{l}\operatorname{HCC}(1) \\
(n=37)\end{array}$ & $\begin{array}{l}\text { CLD (2) } \\
(n=37)\end{array}$ & $\begin{array}{c}\text { Control (3) } \\
(n=36)\end{array}$ & P-value \\
\hline Age $^{\$}$ & $57.24 \pm 9.3$ & $58.87 \pm 9.7$ & $52.50 \pm 8.9$ & \multirow{2}{*}{$=0.369^{*}$} \\
\hline P-value** & $1 \mathrm{vs} 2=0.572$ & $2 \mathrm{vs} 3=0.212$ & $1 \mathrm{vs} 3=0.286$ & \\
\hline Sex & & & & \multirow{4}{*}{$=0.941^{* * *}$} \\
\hline Female & $10(27 \%)$ & 11 (29.7\%) & $11(30.6 \%)$ & \\
\hline Male & 27 (73\%) & $26(70.3 \%)$ & $25(69.4 \%)$ & \\
\hline P-value $\#$ & $1 \mathrm{vs} 2=0.726$ & $2 v s 3=0.941$ & $1 \mathrm{vs} 3=0.749$ & \\
\hline \multicolumn{5}{|l|}{ Occupation } \\
\hline Agricultural (Yes) & $19(51.4 \%)$ & 17 (45.9\%) & $12(33.3 \%)$ & $=0.427^{* * *}$ \\
\hline Housewife (Yes) & $11(29.7 \%)$ & $12(32.4 \%)$ & $14(38.9 \%)$ & $=0.697^{* * *}$ \\
\hline Other Jobs (Yes) & $5(13.5 \%)$ & $5(13.5 \%)$ & $7(19.4 \%)$ & $=0.722^{* * *}$ \\
\hline P-value \# & $1 \mathrm{vs} 2=0.571$ & $2 v s 3=0.389$ & $1 \mathrm{vs} 3=0.778$ & \multirow{4}{*}{$=0.044^{* * *}$} \\
\hline Farming related work & $20(54.1 \%)$ & $10(27 \%)$ & $13(36.1 \%)$ & \\
\hline P-value $\#$ & $1 \mathrm{vs} 2=0.031$ & $2 \mathrm{vs} 3=0.206$ & $1 \mathrm{vs} 3=0.042$ & \\
\hline & & & & \\
\hline Rural & $28(75.7 \%)$ & $29(78.4 \%)$ & $26(72.2 \%)$ & \multirow{3}{*}{$=0.559^{* * *}$} \\
\hline Urban & $9(24.3 \%)$ & $8(21.6 \%)$ & $10(27.8 \%)$ & \\
\hline P-value $\#$ & $1 \mathrm{vs} 2=0.192$ & $2 \mathrm{vs} 3=0.542$ & $1 \mathrm{vs} 3=0.604$ & \\
\hline
\end{tabular}

Table 2. Laboratory findings in the study groups. *Chi-square test was used to compare the percentages between groups. ${ }^{* *}$ Z-test was used to compare the improvement percentages. ${ }^{* * *}$ ANOVA test was used to compare the mean difference between groups. \$Student's t-test was used to compare the mean difference between the two groups

\begin{tabular}{|c|c|c|c|c|}
\hline $\begin{array}{l}\text { Laboratory } \\
\text { findings }\end{array}$ & $\begin{array}{l}\mathrm{HCC}(1) \\
(\mathrm{n}=37)\end{array}$ & $\begin{array}{l}\text { CLD (2) } \\
(n=37)\end{array}$ & $\begin{array}{c}\text { Control (3) } \\
(n=36)\end{array}$ & P-value* \\
\hline ALT & $63.19 \pm 18.5$ & $52.32 \pm 17.6$ & $28.80 \pm 15.5$ & \multirow{2}{*}{$=0.072$} \\
\hline P-value ** & $1 \mathrm{vs} 2=0.482$ & $2 \mathrm{vs} 3=0.119$ & $1 \mathrm{vs} 3=0.025$ & \\
\hline AST & $68.14 \pm 8.9$ & $45.30 \pm 3.2$ & $24.23 \pm 2.6$ & \multirow{2}{*}{$<0.001$} \\
\hline P-value ${ }^{* *}$ & $1 \mathrm{vs} 2=0.004$ & $2 \mathrm{vs} 3=0.008$ & $1 \mathrm{vs} 3<0.001$ & \\
\hline ALP & $152.22 \pm 10.3$ & $154.38 \pm 11.5$ & $79.50 \pm 10.0$ & \multirow{2}{*}{$<0.001$} \\
\hline P-value ** & $1 \mathrm{vs} 2=0.867$ & $2 \mathrm{vs} 3<0.001$ & 1 vs $3<0.001$ & \\
\hline Hemoglobin & $9.91 \pm 2.2$ & $10.08 \pm 1.3$ & $13.77 \pm 1.2$ & \multirow{2}{*}{$<0.001$} \\
\hline P-value ${ }^{* *}$ & $1 \mathrm{vs} 2=0.665$ & $2 \mathrm{vs} 3<0.001$ & 1 vs $3<0.001$ & \\
\hline Platelets & $172.97 \pm 24.7$ & $191.97 \pm 17.9$ & $266.46 \pm 15.2$ & \multirow{2}{*}{$=0.003$} \\
\hline P-value ${ }^{* *}$ & $1 \mathrm{vs} 2=0.459$ & $2 \mathrm{vs} 3=0.009$ & $1 \mathrm{vs} 3=0.001$ & \\
\hline Bilirubin & $31.19 \pm 4.7$ & $29.36 \pm 5.6$ & $10.67 \pm 1.1$ & \multirow{2}{*}{$=0.001$} \\
\hline P-value ** & $1 \mathrm{vs} 2=0.761$ & $2 \mathrm{vs} 3=0.002$ & $1 \mathrm{vs} 3=0.001$ & \\
\hline Albumin & $27.88 \pm 5.5$ & $27.84 \pm 4.4$ & $34.58 \pm 0.7$ & \multirow{2}{*}{$<0.001$} \\
\hline P-value ** & $1 \mathrm{vs} 2=0.970$ & $2 \mathrm{vs} 3<0.001$ & $1 \mathrm{vs} 3<0.001$ & \\
\hline Creatinine & $87.41 \pm 7.1$ & $87.03 \pm 12.9$ & $72.52 \pm 8.3$ & \multirow{2}{*}{$=0.014$} \\
\hline P-value** & $1 \mathrm{vs} 2=0.952$ & $2 \mathrm{vs} 3=0.012$ & $1 \mathrm{vs} 3=0.010$ & \\
\hline
\end{tabular}


Ku86, respectively. Serum vimentin had a higher AUC than AFP, LMNB1, and anti-Ku86 $(P<0.001)$ (Table 9).

Logistic regression analysis of LMNB1, vimentin, and antiKu86 for the diagnosis of HCC determined AUCs of $0.927 \quad(95 \%$ confidence interval [CI] 0.884-0.969), 0.954 (95\% CI $0.919-$ $0.988)$, and $0.933(95 \%$ $\mathrm{CI} \quad 0.886-0.980)$ $(P<0.001)$, respectively (Table 10, Fig. 4). Vimentin mRNA had the highest accuracy (93\%), with sensitivity of $94 \%$, specificity of $92 \%$, positive predictive value (PPV) of 92.2\%, and negative predictive value (NPV) of 93.9\%, followed by anti-Ku86 (accuracy, 87\%; sensitivity, 94\%; specificity, 80\%; PPV, 82.5\%; NPV, 93\%), LMNB1 (accuracy, 80.5\%; sensitivity, 96\%; specificity, 65\%; PPV, 73.3\%; and NPV, 94.2\%), and AFP (accuracy, 77.5\%; sensitivity, 55\%; specificity, 100\%; PPV, 55\%; and NPV, 69\%) (Table 11).

\section{Discussion}

Although HCC is the fifth most common cancer, it is the third highest cause of cancer-related death, with approximately700, 000 deaths per year [1-3]. In recent years, surveillance strategies in patients at a higher risk of HCC have led to the diagnosis of the disease at much earlier stages. Patients in the early stages have a much higher chance of curative response with different treatment options [1]. While surgery is the most effective treatment for liver tumors, about $80 \%$ of HCCs are notcurable at presentation, and therefore the patients die due to the delayed diagnosis. Most cases of HCC arise from prior pathologies,
Table 4. Relationships between tumornumber and tumor markers. *Chi-square test was used to compare the percentages between groups. ${ }^{* *}$ Kruskal-Wallis test was used to compare the median difference between groups

\begin{tabular}{lcccl}
\hline Biomarkers & $\begin{array}{c}\text { Single } \\
(\mathrm{n}=25)\end{array}$ & $\begin{array}{c}\text { Two } \\
(\mathrm{n}=7)\end{array}$ & $\begin{array}{c}\text { Multiple } \\
(\mathrm{n}=5)\end{array}$ & P-value \\
\hline Alpha-fetoprotein & & & & \\
$<20 \mathrm{ng} / \mathrm{ml}$ & $4(14.5 \%)$ & $1(10.5 \%)$ & $1(20 \%)$ & $=0.610^{*}$ \\
$20-200 \mathrm{ng} / \mathrm{ml}$ & $7(29 \%)$ & $4(47.4 \%)$ & $1(20 \%)$ & \\
$>200 \mathrm{ng} / \mathrm{ml}$ & $14(56.5 \%)$ & $2(42.1 \%)$ & $3(60 \%)$ & \\
Lamin B1 & $9.43 \pm 2.9$ & $10.08 \pm 3.8$ & $9.25 \pm 2.6$ & $=0.807^{* *}$ \\
Vimentin & $8.16 \pm 1.6$ & $7.82 \pm 1.7$ & $7.10 \pm 1.5$ & $=0.430^{* *}$ \\
Anti-Ku86 & $0.57 \pm 0.3$ & $0.63 \pm 0.3$ & $0.58 \pm 0.4$ & $=0.889^{* *}$ \\
\hline
\end{tabular}

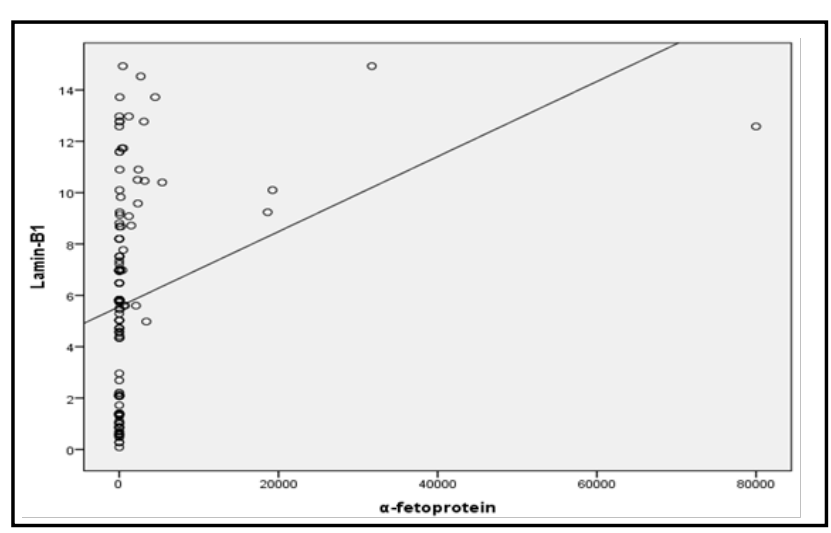

Fig. 2. Correlations between alpha-fetoprotein and lamin B1 levels $(r=0.26, \mathrm{P}=0.034)$.

Table 5. Correlations between tumor size and tumor markers. *Chi-square test was used to compare the percentages between groups. ${ }^{* *}$ Kruskal-Wallis test was used to compare the median difference between groups

\begin{tabular}{lcccc}
\hline Biomarkers & $\begin{array}{c}<2 \mathrm{~cm} \\
(\mathrm{n}=4)\end{array}$ & $\begin{array}{c}2-5 \mathrm{~cm} \\
(\mathrm{n}=13)\end{array}$ & $\begin{array}{c}>5 \mathrm{~cm} \\
(\mathrm{n}=20)\end{array}$ & P-value \\
\hline Alpha-fetoprotein & & & & \\
$<20 \mathrm{ng} / \mathrm{ml}$ & $1(16.7 \%)$ & $2(22.2 \%)$ & $3(6.9 \%)$ & $=0.209^{*}$ \\
$20-200 \mathrm{ng} / \mathrm{ml}$ & $1(16.7 \%)$ & $4(27.8 \%)$ & $7(39.7 \%)$ & \\
$>200 \mathrm{ng} / \mathrm{ml}$ & $2(66.6 \%)$ & $7(50 \%)$ & $10(53.4 \%)$ & \\
Lamin B1 & $12.68 \pm 0.9$ & $8.38 \pm 2.8$ & $10.23 \pm 2.9$ & $=0.014^{* *}$ \\
Vimentin & $6.93 \pm 0.9$ & $8.37 \pm 1.8$ & $7.77 \pm 1.5$ & $=0.198^{* *}$ \\
Anti-Ku86 & $0.57 \pm 0.4$ & $0.59 \pm 0.4$ & $0.59 \pm 0.3$ & $=0.844^{* *}$ \\
\hline
\end{tabular}


so a biomarker for surveillance in high-risk individuals is an essential approach for early detection and improvement of patient outcomes [1]. Serum concentrations of AFP have been shown to be the most useful tumor marker with regards to HCC but levels may be normal in up to $40 \%$ of patients, reducing its sensitivity. Moreover, it may be increased in patients with hepatitis and cirrhosis, compromising its specificity [2527]. A more sensitive serological indicator that distinguishes between HCC and benign hepatic lesions would therefore be very useful for early and specific diagnosis. Hence, the present study was intended to assess circulating vimentin, LMNB1 mRNA, and anti Ku86 as noninvasive indicators for early recognition of HCC, and consequently to evaluate the clinical usefulness and sensitivity of a novel indicator in plasma.

Our results revealed a statistically significant increase in the level of LMNB1in HCC patients compared with CLD patients and HCs. This finding is in accordance with the report of Salway et al. [11], who found that the overexpression of LMNB1 is positively correlated with tumor development. Correspondingly, Sun et al. [28] reported that LMNB1is a potential HCC marker that is increased both intracellularly and extracellularly in the form of protein and mRNA, correspondingly. The expression of this marker could be detected at the earliest stage of HCC. Sun et al. [29] found a significantly higher level of LMNB1 in HCC

patients. In addition, a significantly higher level of LMNB1 was evident in their early HCC patient group than in the cirrhotic group. Similarly, the present study showed that vimentin was significantly increased in HCC compared with CLD and HC groups. These findings are in agreement with those of Sun et al. [28], who stated that vimentin is heavily secreted by small HCCs. Their results were obtained through proteomic analysis to reveal the overexpression of LMNB1, which was strongly associated with an increased number of tumor nodules and 
larger tumor size. Conventional RT-PCR revealed an increase in the positivity rate of circulating LMNB1 mRNA that gradually increased with tumor stage progression. Furthermore, Holmila et al. [30] provided evidence that the methylation levels of vimentin may be an effective biomarker for detecting and monitoring HCC.

Serum levels of anti-Ku86were significantly higher in the HCC patients than in the CLD and HC groups. This finding is in accordance with the results of Nomura et al., [20] which were obtained through proteomic analysis of HCC. Their work provided evidence that antigenicity to Ku86 is developed at a relatively early stage of tumorigenesis. In addition, Nomura et al. [20] reported that the serum anti-Ku86 level is significantly elevated and is unrelated to viral hepatitis B and $\mathrm{C}$ infection in HCC patients. The serum levels of anti-Ku86 may be a potential biomarker for the early detection of HCC and could be used in combination with AFP in clinics. Xu et al. [14] also reported that Ku86 and Ku86 antibodies are promising tumor markers for the early detection and prognosis prediction of

Table 7. Univariate correlations of tumor characteristics with tumor markers in HCC patients $(n=37)$. *Spearman rank correlation coefficient

\begin{tabular}{lcccccc}
\hline \multirow{2}{*}{ Biomarkers } & \multicolumn{2}{c}{ Number } & \multicolumn{2}{c}{ Size } & \multicolumn{2}{c}{ PVT } \\
& rho* $^{*}$ & P-value** & Rho & P-value & rho & P-value \\
\hline Alpha-fetoprotein & -0.07 & $>0.05$ & 0.09 & $>0.05$ & 0.03 & $>0.05$ \\
Lamin B1 & 0.03 & $>0.05$ & 0.14 & $>0.05$ & 0.17 & $>0.05$ \\
Vimentin & -0.18 & $>0.05$ & -0.09 & $>0.05$ & -0.31 & $=0.014$ \\
Anti-KU86 & 0.04 & $>0.05$ & 0.07 & $>0.05$ & 0.10 & $>0.05$ \\
\hline
\end{tabular}

Table 8. Univariate correlations for tumor markers in HCC patients $(\mathrm{n}=37) .{ }^{*}$ Spearman rank correlation coefficient

\begin{tabular}{lcccccc}
\hline \multirow{2}{*}{ Biomarkers } & \multicolumn{2}{c}{ Lamin B1 } & \multicolumn{2}{c}{ Vimentin } & \multicolumn{2}{c}{ Anti-KU86 } \\
& rho* & P-value** & rho & P-value & rho & P-value \\
\hline Alpha-fetoprotein & 0.26 & $=0.034$ & 0.09 & $>0.05$ & 0.04 & $>0.05$ \\
Lamin B1 & 1 & & 0.03 & $>0.05$ & 0.40 & $=0.002$ \\
Vimentin & -0.16 & $>0.05$ & 1 & & -0.21 & $=0.075$ \\
\hline
\end{tabular}

Fig. 5. ROC curve for HCC biomarkers for distinguishing HCC from CLD in the study groups.

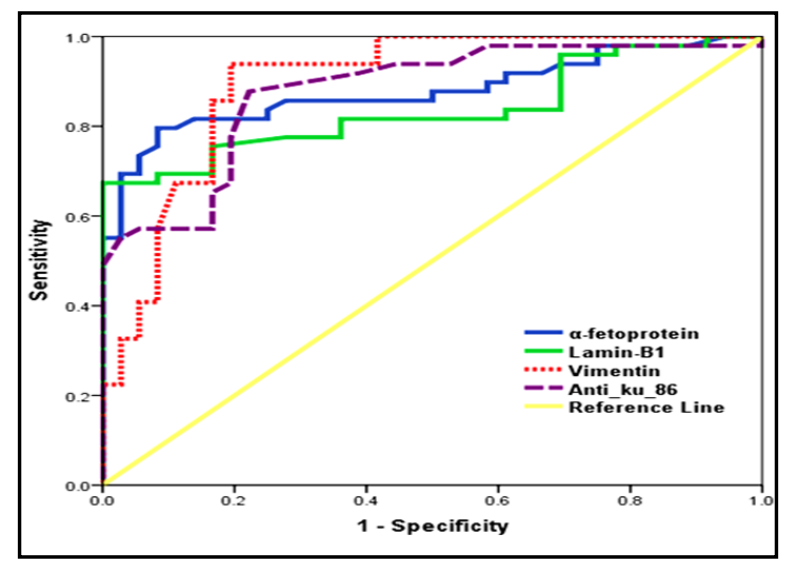

Fig. 6. ROC curve for HCC biomarkers for the study groups.

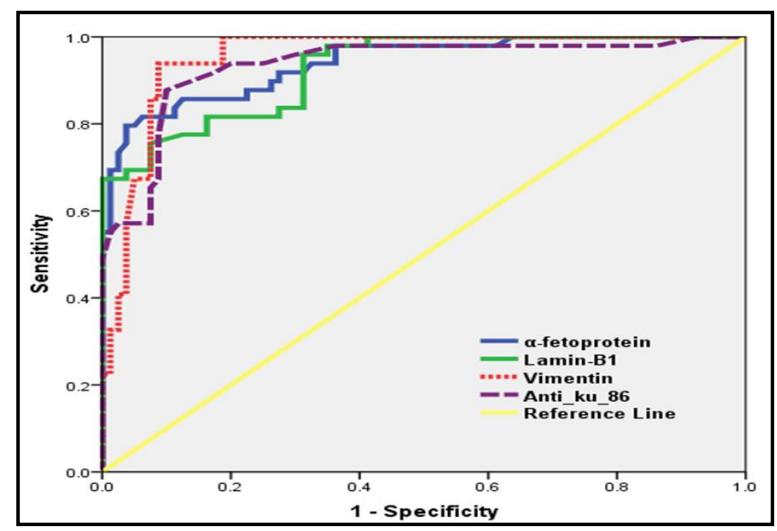


Fig. 7. Relationship between tumor size and serum alpha-fetoprotein cut-offs.

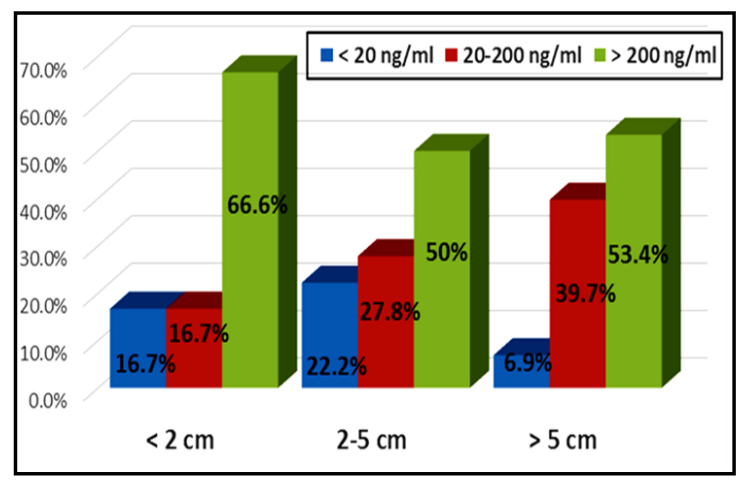

hepatitis $\mathrm{B}$ virus-related HCC. Correlation analysis of tumor size with tumor markers found no significant correlations for AFP, vimentin, and anti-Ku86but surprisingly found that LMNB1 was higher in patients with smaller tumors $<2 \mathrm{~cm}$ than in those with larger tumors. In addition, the vimentin level was found to be decreased with portal vein thrombosis. Similarly, no correlation was found between tumor number and tumor markers. The present study showed a significant correlation of AFP with LMNB1 and of LMNB1 with anti-Ku86, which may boost the early diagnosis of HCC.

\section{Conclusion}

In Conclusion, the clinicopathological and etiological features of HCC represent a considerable challenge for identifying potential biomarkers for use in clinical practice. However, to improve the diagnostic accuracy of tests for HCC, new biomarkers that can be combined with AFP are desired, particularly because many patients with benign liver diseases also have elevated serum AFP. An increase in the circulating levels of antiKu86, LMNB1, and vimentin could be a possible replacement marker, either alone or in combination with AFP. Although vimentin had a higher AUC than serum AFP, the combination
Table 9. Diagnostic performance of cancer biomarkers for distinguishing HCC from CLD. AUC $=$ area under the curve, $\mathrm{SE}=$ Standard Error, $\mathrm{CI}=$ Confidence Interval, ${ }^{*}$ Null hypothesis: true area $=0.5$

\begin{tabular}{lcccc}
\hline Biomarkers & AUC & $95 \%$ CI & SE & P-value* \\
\hline Alpha-fetoprotein & 0.878 & $0.804-0.953$ & 0.038 & $<0.001$ \\
Lamin B1 & 0.837 & $0.751-0.923$ & 0.044 & $<0.001$ \\
Vimentin & 0.897 & $0.825-0.969$ & 0.037 & $<0.001$ \\
Anti_KU86 & 0.876 & $0.802-0.950$ & 0.038 & $<0.001$ \\
\hline
\end{tabular}

Table 10. Diagnostic performance of cancer biomarkers for distinguishing HCC. AUC = Area under the Curve, SE = Standard Error, $\mathrm{CI}=$ Confidence Interval, ${ }^{*}$ Null hypothesis: true area $=0.5$

\begin{tabular}{lcccc}
\hline \multirow{2}{*}{ Parameter } & \multicolumn{4}{c}{ Diagnostic accuracy measures } \\
& $\alpha$-fetoprotein & Lamin B1 & Vimentin & Anti-KU86 \\
\hline AUC & 0.940 & 0.927 & 0.954 & 0.933 \\
Cut-off & 100 & 5.16 & 5.86 & 0.245 \\
Accuracy & $77.5 \%$ & $80.5 \%$ & $93 \%$ & $87 \%$ \\
Sensitivity, $\%$ & $55 \%$ & $96 \%$ & $94 \%$ & $94 \%$ \\
Specificity, \% & $100 \%$ & $65 \%$ & $92 \%$ & $80 \%$ \\
PPV, \% & $55 \%$ & $73.3 \%$ & $92.2 \%$ & $82.5 \%$ \\
NPV, $\%$ & $69 \%$ & $94.2 \%$ & $93.9 \%$ & $93 \%$ \\
\hline
\end{tabular}

Table 11. Diagnostic accuracy measuresof biomarkers for the diagnosis of HCC in the studied sample. *Sensitivity (true positives/all diseased); specificity (true negatives/all non-diseased); PPV (true positives/all test positives); NPV (true negatives/all test negatives)

\begin{tabular}{lcccc}
\hline \multirow{2}{*}{ Parameter } & \multicolumn{4}{c}{ Diagnostic accuracy measures } \\
& $\alpha$-fetoprotein & Lamin B1 & Vimentin & Anti-KU86 \\
\hline AUC & 0.940 & 0.927 & 0.954 & 0.933 \\
Cut-off & 100 & 5.16 & 5.86 & 0.245 \\
Accuracy & $77.5 \%$ & $80.5 \%$ & $93 \%$ & $87 \%$ \\
Sensitivity, \% & $55 \%$ & $96 \%$ & $94 \%$ & $94 \%$ \\
Specificity, \% & $100 \%$ & $65 \%$ & $92 \%$ & $80 \%$ \\
PPV, \% & $55 \%$ & $73.3 \%$ & $92.2 \%$ & $82.5 \%$ \\
NPV, \% & $69 \%$ & $94.2 \%$ & $93.9 \%$ & $93 \%$ \\
\hline
\end{tabular}


of vimentin and AFP further improved the sensitivity and specificity. Thus, vimentin might be an important independent and discriminative serological biomarker for detecting earlystage HCC in patients with normal serum AFP. Anti-Ku86 and LMNB1levels can be accurately measured in current commercial tests and could significantly improve our ability to identify such patients and thereby allow them to receive earlier treatment. Taken together, these results imply that these biomarkers could be essential tools for cancer therapy in the treatment of HCC.

\section{Abbreviations}

AFP (alpha-fetoprotein); AU (area under the curve); CHB (chronic hepatitis B); CHC (chronic hepatitis C); CLD (chronic liver disease); HCC (hepatocellular carcinoma); ROC (receiver operating characteristic); RT-PCR (real-time polymerase chain reaction).

\section{Acknowledgements}

N.K. Idriss, performed the molecular measurements, interpreting the results, designed the figures and worked on the manuscript. M.F. processed the data and drafted the manuscript. H.I. and F.A. were involved in planning and revising the work. L.A.,W.A.,M.A.,Z.S.,A.M.,H.A. were responsible for patients recuritments calculations, interpreting the results and worked on the manuscript. All authors discussed the results, commented on the manuscript, conceiving, designing analysis and writing of the paper.

\section{Disclosure Statement}

The authors declare no conflicts of interest.

\section{References}

- 1 Black AP, Mehta AS: The search for biomarkers of hepatocellular carcinoma and the impact on patient outcome. Curr Opin Pharmacol 2018;41:74-78.

2 Ghouri YA, Mian I, Rowe JH: Review of hepatocellular carcinoma: Epidemiology, etiology, and carcinogenesis. J Carcinog 2017;16:1.

- 3 Zhu RX, Seto WK, Lai CL, Yuen MF: Epidemiology of Hepatocellular Carcinoma in the Asia-Pacific Region. Gut Liver 2016;10:332-339.

- 4 El-Serag HB: Epidemiology of viral hepatitis and hepatocellular carcinoma. Gastroenterology 2012;142:1264-1273.e1261.

- 5 el-Zayadi AR, Badran HM, Barakat EM, Attia Mel D, Shawky S, Mohamed MK, Selim O, Saeid A: Hepatocellular carcinoma in Egypt: a single center study over a decade. World J Gastroenterol 2005;11:5193-5198.

- 6 Singal AG, Conjeevaram HS, Volk ML, Fu S, Fontana RJ, Askari F, Su GL, Lok AS, Marrero JA: Effectiveness of hepatocellular carcinoma surveillance in patients with cirrhosis. Cancer Epidemiol Biomarkers Prev 2012;21:793-799.

7 Shu H, Li W, Shang S, Qin X, Zhang S, Liu Y: Diagnosis of AFP-negative early-stage hepatocellular carcinoma using Fuc-PON1. Discov Med 2017;23:163-168.

8 Moir RD, Yoon M, Khuon S, Goldman RD: Nuclear lamins A and B1: different pathways of assembly during nuclear envelope formation in living cells. J Cell Biol 2000;151:1155-1168.

- 9 Hutchison CJ: B-type lamins in health and disease. Semin Cell Dev Biol 2014;29:158-163.

- 10 Elcock LS, Bridger JM: Exploring the relationship between interphase gene positioning, transcriptional regulation and the nuclear matrix. Biochem Soc Trans 2010;38:263-267. 


\section{Cellular Physiology Cell Physiol Biochem 2019;52:595-605

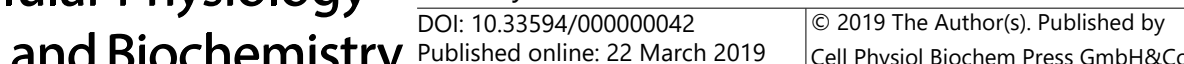 and Biochemistry Published online: 22 March 2019 Cell Physiol Biochem Press GmbH\&Co. KG \\ Idriss et al.: Genetic Indices in Patients with Hepatocellular Carcinoma}

- 11 Salway F, Day PJ, Ollier WE, Peakman TC: Levels of 5' RNA tags in plasma and buffy coat from EDTA blood increase with time. Int J Epidemiol 2008;37:i11-15.

- 12 Shimi T, Butin-Israeli V, Adam SA, Hamanaka RB, Goldman AE, Lucas CA, Shumaker DK, Kosak ST, Chandel NS, Goldman RD: The role of nuclear lamin B1 in cell proliferation and senescence. Genes Dev 2011;25:2579-2593.

- 13 AlSalloom AA: An update of biochemical markers of hepatocellular carcinoma. Int J Health Sci (Qassim) 2016;10:121-136.

- 14 Xu Y, Liu AJ, Gao YX, Hu MG, Zhao GD, Zhao ZM, Liu R: Expression of Ku86 and presence of Ku86 antibody as biomarkers of hepatitis B virus related hepatocellular carcinoma. Dig Dis Sci 2014;59:614-622.

- 15 Liu L, Wang J, Shi L, Zhang W, Du X, Wang Z, Zhang Y: beta-Asarone induces senescence in colorectal cancer cells by inducing lamin B1 expression. Phytomedicine 2013;20:512-520.

- 16 Roggiani M, Goulian M: Chromosome-Membrane Interactions in Bacteria. Annu Rev Genet 2015;49:115129.

- 17 Ogrodnik M, Salmonowicz H, Brown R, Turkowska J, Sredniawa W, Pattabiraman S, Amen T, Abraham AC, Eichler N, Lyakhovetsky R, Kaganovich D: Dynamic JUNQ inclusion bodies are asymmetrically inherited in mammalian cell lines through the asymmetric partitioning of vimentin. Proc Natl Acad Sci U S A 2014;111:8049-8054.

- 18 Satelli A, Li S: Vimentin in cancer and its potential as a molecular target for cancer therapy. Cell Mol Life Sci 2011;68:3033-3046.

- 19 Lahat G, Zhu QS, Huang KL, Wang S, Bolshakov S, Liu J, Torres K, Langley RR, Lazar AJ, Hung MC, Lev D: Vimentin is a novel anti-cancer therapeutic target; insights from in vitro and in vivo mice xenograft studies. PLoS One 2010;5:e10105.

20 Nomura F, Sogawa K, Noda K, Seimiya M, Matsushita K, Miura T, Tomonaga T, Yoshitomi H, Imazeki F, Takizawa H, Mogushi K, Miyazaki M, Yokosuka O: Serum anti-Ku86 is a potential biomarker for early detection of hepatitis $\mathrm{C}$ virus-related hepatocellular carcinoma. Biochem Biophys Res Commun 2012;421:837-843.

21 Luk JM, Su YC, Lam SC, Lee CK, Hu MY, He QY, Lau GK, Wong FW, Fan ST: Proteomic identification of Ku70/ Ku80 autoantigen recognized by monoclonal antibody against hepatocellular carcinoma. Proteomics 2005;5:1980-1986.

22 Liu CJ, Chen BF, Chen PJ, Lai MY, Huang WL, Kao JH, Chen DS: Role of hepatitis B virus precore/core promoter mutations and serum viral load on noncirrhotic hepatocellular carcinoma: a case-control study. J Infect Dis 2006;194:594-599.

- 23 Caron M, Choquet-Kastylevsky G, Joubert-Caron R: Cancer immunomics using autoantibody signatures for biomarker discovery. Mol Cell Proteomics 2007;6:1115-1122.

- 24 Subramaniam S, Kelley RK, Venook AP: A review of hepatocellular carcinoma (HCC) staging systems. Chin Clin Oncol 2013;2:33.

- 25 Lersritwimanmaen P, Nimanong S: Hepatocellular Carcinoma Surveillance: Benefit of Serum Alfafetoprotein in Real-world Practice. Euroasian J Hepatogastroenterol 2018;8:83-87.

- 26 Yu SJ: A concise review of updated guidelines regarding the management of hepatocellular carcinoma around the world: 2010-2016. Clin Mol Hepatol 2016;22:7-17.

- 27 Massarweh NN, El-Serag HB: Epidemiology of Hepatocellular Carcinoma and Intrahepatic Cholangiocarcinoma. Cancer Control 2017;24:1073274817729245.

28 Sun S, Xu MZ, Poon RT, Day PJ, Luk JM: Circulating Lamin B1 (LMNB1) biomarker detects early stages of liver cancer in patients. J Proteome Res 2010;9:70-78.

29 Sun S, Poon RT, Lee NP, Yeung C, Chan KL, Ng IO, Day PJ, Luk JM: Proteomics of hepatocellular carcinoma: serum vimentin as a surrogate marker for small tumors (<or=2 cm). J Proteome Res 2010;9:1923-1930.

30 Holmila R, Sklias A, Muller DC, Degli Esposti D, Guilloreau P, McKay J, Sangrajrang S, Srivatanakul P, Hainaut P, Merle P, Herceg Z, Nogueira da Costa A: Targeted deep sequencing of plasma circulating cell-free DNA reveals Vimentin and Fibulin 1 as potential epigenetic biomarkers for hepatocellular carcinoma. PLoS One 2017;12:e0174265. 